\title{
Influência do Voleibol na Densidade Mineral Ossea de Adolescentes do Sexo Feminino
}

\section{Influence of Volleyball in the Bone Mineral Density of Female Adolescents}



Wisley Gontijo de Mesquita

Rômulo Maia Carlos Fonseca'

Nancí Maria de França ${ }^{2}$

1. Mestre em Educação Física pela Universidade Católica de Brasília, Núcleo de Estudos em Escolares. 2. Professora Doutora do Programa de Pós-Graduação Stricto Sensu em Educação Física da Universidade Católica de Brasília, Núcleo de Estudos em Escolares.

\section{Endereço para correspondência:} Profa.Dra. Nancí Maria de França. QS 07 Lote 1 EPCT - sala G-119 - 70790-160 - Taguatinga, DF - Brasil.

E-mail: nfranca@ucb.br

Submetido em 26/07/2007 Versão final recebida em 04/04/2008 Aceito em 05/07/2008

\begin{abstract}
RESUMO
Sabe-se que esportes com grandes cargas mecânicas e maior impacto do corpo com o solo resultam em maior massa óssea do que atividades em que o peso do corpo é pouco solicitado. Apesar de o voleibol estar entre os esportes considerados de alto impacto, existem poucos estudos que relacionam sua prática ao desenvolvimento da densidade mineral óssea (DMO). Portanto, o presente estudo teve como objetivo comparar a DMO entre garotas participantes de equipes de treinamento de voleibol e de garotas participantes de atividade física escolar. Foram avaliadas 60 voluntárias com idade entre 13 e 17 anos. Elas foram divididas em grupo voleibol (GV) - composto pelas praticantes de voleibol - e grupo controle (GC). Foram mensurados massa corporal, estatura, consumo de cálcio, DMO do corpo inteiro, da cabeça, do colo do fêmur e do triângulo de Wards e estágio de maturação sexual. Foi utilizado o teste $t$ para amostras independentes, para comparar a diferença entre as médias dos dois grupos $(p<0,05)$. Os resultados mostraram que a DMO do corpo inteiro $(1,174 \pm 0,065)$, colo do fêmur $(1,164 \pm 0,096)$ e triangulo de Wards $(1,111 \pm 0,138)$ do GV é significativamente maior que a DMO do GC (corpo inteiro: 1,083 \pm 0,082; colo do fêmur: 0,998 \pm 0,142; triangulo de Wards: 0,944 $\pm 0,178)$. Porém, a DMO da cabeça não apresentou nenhuma diferença significativa entre os dois grupos. Portanto, a prática de voleibol apresenta-se como importante esporte para a aquisição de massa óssea durante o final da adolescência e a DMO da cabeça como um possível padrão interno de controle da amostra.
\end{abstract}

Palavras-chave: massa óssea, esporte, adolescência.

\begin{abstract}
Several studies have shown that high impact weight-bearing sports are more beneficial than non-weightbearing ones. Despite being considered a high impact sport, few studies relate it with bone mineral density (BMD) development. Therefore, the aim of this study was to compare BMD between female volleyball players and 60 volunteer girls aged 13-17-y who participated in this study. They were classified in Volleyball Group (VG) - who were practicing volleyball - and Control Group (CG). Body mass, height, calcium uptake, total body, head, femoral neck and Wards Triangle BMD and sexual maturation were measured. Differences between groups were analyzed by t-test for independent samples $(p<0.05)$. The VG was statistically higher in total body (1.174 \pm 0.065$)$, femoral neck (1.164 \pm 0.096$)$ and Wards triangle (1.111 \pm 0.138$)$ BMD than CG (total body: $1.083 \pm 0.082$; femoral neck: $0.998 \pm 0.142$; Wards triangle: $0.944 \pm 0.178)$; however, head BMD was not different between groups. Therefore, volleyball practice is an important sport to promote bone mass acquisition during adolescence and head BMD became a possible internal standard for selection of bias control.
\end{abstract}

Keywords: bone mass, sport, adolescence.

\section{INTRODUÇÃO}

Já é conhecido que pelo menos 90\% do pico de massa óssea são adquiridos até os 18 anos de idade ${ }^{(1)}$; fatores genéticos ${ }^{(2)}$, maturacionais ${ }^{(3)}$ e comportamentais ${ }^{(1,4)}$ (ex:: atividade física, alimentação) são os principais determinantes desses ganhos.

Os fatores genéticos são os principais responsáveis pela variação da massa óssea, pois estudos realizados com pares de pais e filhos ${ }^{(2)}$ e com gêmeos ${ }^{(5)}$ demonstraram que a hereditariedade é responsável por variação de 60 a $80 \%$ na determinação da massa óssea. Além disso, durante a adolescência o desenvolvimento da densidade mineral óssea (DMO) está mais associada ao estágio da maturação sexual do que à idade cronológica, pois a DMO não difere entre meninos e meninas antes da puberdade ${ }^{(6)}$. Essa diferenciação na aquisição de massa óssea passa a ocorrer durante a puberdade, pois a mesma é maior durante as idades de 11 a 14 anos para garotas e 13 a 17 para garotos ${ }^{(3)}$.

Dos fatores comportamentais, a alimentação e a atividade física possuem grande importância na otimização do ganho de massa óssea, pois aproximadamente $99 \%$ do cálcio corporal estão depositados no esqueleto ${ }^{7}$ e deficiências na ingestão e absorção desse mineral podem comprometer a saúde óssea. Já a relação entre a DMO e a atividade física pode ser demonstrada pela perda substancial do osso, devido a imobilização prolongada; pacientes imobilizados podem perder $40 \%$ da massa óssea original em um ano(8).

A adaptação óssea decorrente da atividade física está relacionada com a intensidade da tensão no osso e a adaptação desse osso ao estímulo(9). Quando a atividade está abaixo dos valores fisiológicos mínimos de tensão, ocorre a perda da massa óssea. Dentro da zona de carga fisiológica, o osso é mantido; os ganhos ocorrerão somente 
quando a intensidade de carga for aumentada. Em resposta a cargas extremas, um novo osso desorganizado pode ser produzido.

Dessa forma, alguns estudos demonstraram que esportes com grandes cargas mecânicas e maior impacto do corpo com o solo resultam em maior massa óssea do que atividades em que o peso do corpo é pouco solicitado ${ }^{(10,11)}$. Entre os diversos esportes, o voleibol é caracterizado pela presença de alta exigência muscular e da ação das forças gravitacionais agindo sobre o esqueleto durante as mudanças de direção, paradas, saltos e bloqueios. Há produção de forças que varia de três a seis vezes o peso corporal, sendo classificado como um esporte de alto impacto ${ }^{(12)}$.

Apesar de o voleibol estar entre os esportes considerados de alto impacto, existem poucos estudos que relacionam sua prática ao desenvolvimento da $\mathrm{DMO}^{(13-15)}$. Portanto, o presente estudo teve como objetivo comparar a DMO entre garotas participantes de equipes de treinamento de voleibol e de garotas participantes de atividade física escolar.

\section{MÉTODOS}

\section{Amostra}

Foram avaliadas 60 voluntárias selecionadas em escolas e clubes nas cidades de Anápolis, Inhumas e Goiânia-GO, com idade entre 13 e 17 anos. Essas adolescentes foram divididas em dois grupos, voleibol (GV) e controle (GC). O GV foi composto pelas praticantes de voleibol que participavam havia mais de um ano em equipes de competição de suas escolas e o GC, por praticantes de atividade física escolar que não participavam de exercícios físicos regulares fora escola havia mais de um ano.

Os critérios de inclusão adotados foram: estar nos estágios IV ou $\checkmark$ de maturação sexual segundo os critérios de Tanner; estar regularmente matriculada e freqüentando a escola; não possuir doença crônico-degenerativa, nem histórico de doenças que afetassem o desenvolvimento ósseo e que comprometessem a prática de exercícios físicos. Para a inclusão no GV, os seguintes critérios também foram adotados: participar de pelo menos 75\% das sessões de treinamentos de voleibol de suas equipes; praticar voleibol há mais de três anos; integrar a equipe de competição de suas escolas há mais de um ano; participar de equipes que tinham carga horária de treinamento entre 270 e 360 minutos semanais.

Todas as participantes e seus responsáveis preencheram e assinaram um termo de consentimento livre e esclarecido, além disso, também apresentaram autorização para tráfego até Brasília expedido por Juizado de Menores. O estudo foi aprovado pelo Comitê de Ética em Pesquisa da Universidade Católica de Brasília (CEP/UCB No 022/2004), de acordo com a Resolução 196/96.

\section{PROCEDIMENTOS}

Para caracterizar a população avaliada e selecionar as participantes, conforme os critérios de inclusão, as adolescentes responderam a uma anamnese de saúde e um questionário recordatório da dieta alimentar. Após as devidas verificações, elas foram conduzidas ao Laboratório de Imagens da Universidade Católica de Brasília para as avaliações antropométricas e os exames de densitometria óssea.

\section{Anamnese de saúde, recordatório de dispêndio energético e alimentar}

A anamnese de saúde utilizada no protocolo do Núcleo de Estudos em Escolares/UCB foi adaptada para os objetivos e critérios do presente estudo. Nesse sentido, foram acrescidas algumas perguntas sobre o histórico de participação no voleibol e sobre o histórico menstrual. Para o dispêndio energético, foi utilizado o recordatório de três dias conforme apresentado por Giugliano ${ }^{(16)}$. Também foi aplicado um recordatório alimentar de um dia para avaliar a dieta alimentar e o consumo de cálcio. Para a análise da ingestão calórica e consumo de cálcio, foi utilizada a tabela para a avaliação de consumo alimentar em medidas caseiras, descrita por Pinheiro et al. ${ }^{(17)}$.

\section{Antropometria}

A massa corporal (kg) e a estatura (m) foram avaliadas por equipamentos padronizados. A estatura foi mensurada com um estadiômetro, da marca Seca, fixado à parede com resolução de 0,1cm, e a massa corporal foi medida em uma balança digital com resolução de $100 \mathrm{~g}$ da marca Filizola. $\mathrm{O}$ índice de massa corporal (IMC) foi obtido pela fórmula: massa corporal $(\mathrm{kg}) \div \operatorname{estatura}^{2}(\mathrm{~m})$.

\section{Densidade mineral óssea e composição corporal}

A DMO do corpo inteiro $(C l)$, do colo do fêmur (CF) e triângulo de Wards (TW) foram mensurados por um aparelho de absortometria de raios $X$ de dupla energia (DXA), da marca Lunar, modelo DPX-IQ (software versão 4.7e). A DMO da cabeça (crânio) foi determinada pelo resultado obtido no exame do corpo inteiro. A precisão do aparelho utilizado foi previamente discutida na literatura(18), mas para avaliar a variação (desvio-padrão) de cada sítio ósseo mensurado em nosso laboratório, uma pessoa foi avaliada oito vezes durante oito dias; a variância encontrada para a DMO de todos os sítios situou-se entre 0,07\% e 2,4 \%. A massa corporal magra (MCM) e gordura corporal (GC) foram determinadas pela mensuração do corpo inteiro; a variância encontrada entre as avaliações repetidas foi de 1,9\% e 2,1\%, respectivamente. A calibragem do aparelho era realizada diariamente, conforme as normas do fabricante e todos os exames foram analisados pelo mesmo técnico.

\section{Maturação sexual}

O estágio puberal foi determinado por auto-avaliação de seios e pêlos pubianos, um método de reconhecida validade e confiança ${ }^{(19)}$. Por haver diferenças na aquisição de massa óssea durante os cinco estágios de maturação sexual, foram escolhidos os estágios IV e V para controlar a influência maturacional sobre a DMO das adolescentes.

\section{Análise estatística}

As variáveis foram analisadas de forma descritiva e inferencial por meio de médias e desvios-padrão e teste $t$ para amostras independentes, para comparar as médias dos dois grupos; o nível de significância adotado foi de $p \leq 0,05$. Foi utilizado o pacote estatístico SPSS para Windows, versão 10.0 .

\section{RESULTADOS}

As médias e os desvios-padrão da idade, variáveis antropométricas, dispêndio energético, ingestão calórica e consumo de cálcio diário nos dois grupos estão apresentados na tabela 1.

No grupo de praticantes de voleibol (GV), observou-se freqüência de três sessões semanais, com duração entre 90 e 120 minutos por sessão, totalizando carga horária média de 299,3 \pm 39,9 minutos de treinamento por semana. A maioria dessas garotas já participava de treinamentos havia três ou quatro anos; algumas delas já praticavam havia mais de quatro anos. As principais competições disputadas pelo GV eram jogos escolares e regionais. 
Tabela 1. Idade e características das participantes.

\begin{tabular}{|c|c|c|}
\hline & $\begin{array}{c}G C \\
(N=30)\end{array}$ & $\begin{array}{c}\text { GV } \\
(\mathrm{N}=30)\end{array}$ \\
\hline Idade (anos) & $15,53 \pm 0,97^{*}$ & $14,63 \pm 1,06$ \\
\hline Menarca (anos) & $12,0 \pm 0,94$ & $11,57 \pm 1,22$ \\
\hline Massa corporal (Kg) & $51,68 \pm 8,34$ & $58,31 \pm 9,55^{*}$ \\
\hline Estatura $(\mathrm{cm})$ & $158,4 \pm 5,88$ & $164 \pm 8,18^{*}$ \\
\hline IMC $\left(\mathrm{Kg} / \mathrm{m}^{2}\right)$ & $20,58 \pm 3,15$ & $21,60 \pm 2,56$ \\
\hline Massa corporal magra (Kg) & $34,26 \pm 3,61$ & $40,15 \pm 4,87^{*}$ \\
\hline Gordura corporal (\%) & $29,69 \pm 7,77$ & $27,82 \pm 7,00$ \\
\hline Gasto calórico diário (kcal/Kg/dia) & $38,07 \pm 3,44$ & $42,53 \pm 3,54^{*}$ \\
\hline Ingestão calórica diária (Kcal/dia) & $1856,9 \pm 316,9$ & $2076,7 \pm 588,2$ \\
\hline Consumo de Cálcio (mg/dia) & $476,4 \pm 230$ & $613,7 \pm 227$ \\
\hline
\end{tabular}

${ }^{*} p \leq 0,05$ - Diferença estatisticamente significativa em relação ao outro grupo (média \pm desvio padrão)

Em relação às participantes do grupo controle (GC), todas estudavam em escolas que ministravam aulas de Educação Física inseridas na grade horária comum a todas as matérias. A grande maioria (79,6\%) nunca participou de atividades físicas orientadas fora da escola ou mesmo em turno contrário ao das aulas; as que já tinham participado de atividades físicas regulares extra-escolares não o faziam havaia no mínimo dois anos. Foram verificadas diferenças significativas entre os grupos nas variáveis massa corporal, estatura e massa magra $(p<0,05)$.

O resultado das comparações entre as médias da DMO do corpo inteiro-Cl, colo do fêmur-CF, triangulo de Ward-TW e da cabeça nos dois grupos encontra-se na tabela 2. Foi observada ocorrência de diferenças significativas em todos os sítios analisados ( $p<0,05)$, exceto para a DMO da cabeça.

Tabela 2. Comparação entre a densidade mineral óssea dos dois grupos.

\begin{tabular}{l|c|c|c}
\hline & $\begin{array}{c}\text { GC } \\
(\mathbf{N}=\mathbf{3 0})\end{array}$ & $\begin{array}{c}\text { GV } \\
(\mathbf{N}=\mathbf{3 0})\end{array}$ & $\begin{array}{c}\text { Valor } \\
\mathbf{t}\end{array}$ \\
\hline $\mathrm{DMO} \mathrm{Cl} \mathrm{(g/ \textrm {cm } ^ { 2 } )}$ & $1,083 \pm 0,082$ & $1,174 \pm 0,065^{*}$ & 4,698 \\
\hline $\mathrm{DMO} \mathrm{CF}\left(\mathrm{g} / \mathrm{cm}^{2}\right)$ & $0,998 \pm 0,142$ & $1,164 \pm 0,096^{*}$ & 5,285 \\
\hline DMO TW $\left(\mathrm{g} / \mathrm{cm}^{2}\right)$ & $0,944 \pm 0,178$ & $1,111 \pm 0,138^{*}$ & 4,054 \\
\hline DMO Cabeça $\left(\mathrm{g} / \mathrm{cm}^{2}\right)$ & $2,063 \pm 0,195$ & $2,099 \pm 0,169$ & 0,775 \\
\hline
\end{tabular}

DMO: densidade mineral óssea; Cl: corpo inteiro; CF: colo do fêmur; TW: Triangulo de Wards. ${ }^{*} p \leq 0,05$ - Diferença estatisticamente significativa em relação ao outro grupo (média \pm desvio padrão)

\section{DISCUSSÃO}

No intuito de esclarecer e entender o efeito dos esportes na aquisição de massa óssea, esse estudo verificou se a prática do voleibol poderia ser um fator relevante para aumentar a massa óssea de garotas com idade entre os 13 e os 17 anos. Porém, por ser um estudo trans- versal, apresentou limitações que devem ser levadas em consideração na analise dos resultados. A avaliação do aspecto nutricional apresenta-se como uma limitação do estudo, pois o acompanhamento mais detalhado da dieta alimentar durante maior período de tempo seria mais eficaz no entendimento da relação entre a quantidade de cálcio ingerido e o voleibol na DMO.

Outra limitação presente no estudo foi a impossibilidade de controlar a regulação hormonal nos grupos avaliados, tendo em vista que a mesma é um fator que pode alterar o pico de massa óssea durante a prática desportiva ${ }^{(20)}$. Porém, como medida para atenuar essa limitação, participaram do estudo somente garotas dos mesmos estágios de maturação sexual (IV-V); também, não foi relatada pelas participantes a presença de qualquer distúrbio menstrual, aumentando a confiabilidade dos dados.

O GC apresentou valores médios de estatura e massa corporal similar aos de dois estudos realizados com escolares da mesma faixa etária em Brasília(21,22) e um realizado em Santa Catarina(23). Já o GV, embora tenha a massa corporal similar à de outras jogadoras de voleibol de Curitiba ${ }^{(24)}$ e Brasília(21) e ligeiramente inferior à de jogadoras do Rio de Janeiro(25), também apresentou estatura inferior à dos estudos mencionados acima. Fato que pode ser entendido por esses estudos terem sido realizados com adolescentes das seleções de base de times profissionais de voleibol, diferentemente das participantes do presente estudo.

O percentual de gordura encontrado nos dois grupos foi bem superior ao encontrado em outro estudo(23), porém, quando comparado com estudos que também utilizaram a DXA para avaliar a composição corporal, nossos resultados foram similares ${ }^{(12,26)}$. A densitometria óssea é considerada um método de referencia para a avaliação da composição corporal(27), porém, como o numero de estudos realizados com a densitometria óssea no Brasil é reduzido, torna-se difícil a generalização de nossos resultados.

Tanto o GC quanto o GV apresentaram consumo diário de cálcio muito abaixo do que é recomendado para adolescentes e adultos jovens, que é de $1.200-1.500 \mathrm{mg}$ de cálcio por dia ${ }^{(28)}$. O consumo médio apresentado foi de 476,4mg/dia para o GC e 613,7mg/dia para o GV, o que corresponde a aproximadamente 39,7\% e 51,1\% das recomendações diárias para essa faixa etária, respectivamente. Porém, mesmo com a avaliação da dieta alimentar sendo baseada na avaliação de um dia, nossos resultados estão de acordo com outros estudos que mostraram baixa ingestão de cálcio em adolescentes nessa faixa etária ${ }^{(29-31)}$. Outras jogadoras de voleibol com a mesma faixa etária do Estado do Rio de Janeiro ${ }^{(25)}$ apresentaram maior ingestão calórica (3.945 \pm 633 kcal) e o consumo médio de cálcio de $1.204 \mathrm{mg} /$ dia, correspondendo quase ao dobro do GV avaliado nesse estudo. O consumo de cálcio abaixo do que é recomendado é um fator preocupante tanto para a massa óssea quanto para a própria saúde das adolescentes. Porém, por não terem sido encontradas diferenças significativas no consumo de cálcio dos dois grupos, os resultados encontrados reforçam a importância do esporte no aumento da DMO.

A DMO do Cl, CF e TW no GV foi estatisticamente superior à DMO do GC. Outros estudos realizados com jogadoras de voleibol também demonstraram superioridade na DMO em relação ao grupo controle quando comparados com outros esportes caracterizados como sendo de baixo impacto ${ }^{(12-15)}$. Essa superioridade encontrada no GV pode ser explicada pelo aumento da carga muscular e mecânica exercida pelo voleibol no tecido ósseo, passando dos limites da zona de carga fisiológica ${ }^{(9)}$. Além disso, essas sobrecargas impos- 
tas ao osso produzem tensões de certa magnitude que causam microfraturas ao longo do local onde ela foi gerada, fazendo com que ocorra a osteogênese: os osteoclastos iniciam a remoção do material danificado para que os osteoblastos possam depositar nova matriz mineral óssea ${ }^{(32)}$.

Portanto. a prática desportiva apresenta-se como importante método para otimizar o ganho de massa óssea durante a adolescência. Sabendo que a fratura do fêmur proximal é provavelmente a manifestação mais devastadora da osteoporose ${ }^{(33)}$, e associando os resultados desses estudos aos encontrados na presente pesquisa, podemos inferir que o voleibol é um esporte recomendado para a otimização do pico de massa óssea, particularmente nos sítios do $\mathrm{Cl}$ e fêmur proximal (CF e TW), diminuindo os riscos de osteoporose nesses sítios.

A DMO da cabeça parece não sofrer influência da atividade física, pois estudos realizados com atletas e controle, incluindo o presente estudo, também não encontraram diferenças significativas na DMO da cabeça entre grupo controle e experimental, tanto para o sexo masculino quanto para o feminino ${ }^{(33-35)}$. Isso indica que a sobrecarga de atividades físicas não interfere nos valores de DMO da cabeça, tornando-a um padrão interno de controle de viés no momento da seleção da amostra, conforme sugerido por Nordstrom et al. ${ }^{(33)}$; indivíduos mais fortes costumam participar com mais freqüência das atividades esportivas, o que pode causar viés de seleção da amostra quando se investiga o efeito da atividade física na massa óssea em estudos transversais.

Em resumo, dos resultados obtidos no presente estudo, podemos concluir que a sobrecarga muscular e mecânica exercida no tecido ósseo pela prática do voleibol proporciona maior DMO de Cl, CF e TW em garotas com idade entre 13 e 17 anos quando comparado com aquelas que participam somente da Educação Física escolar. Além disso, a DMO da cabeça apresenta-se como um importante padrão interno de controle da amostra, porém. a realização de estudos de intervenção podem ser mais esclarecedores no entendimento do efeito do voleibol na DMO da cabeça e outros sítios ósseos.

Todos os autores declararam não haver qualquer potencial conflito de interesses referente a este artigo.

\section{REFERÊNCIAS BIBLIOGRÁFICAS}

1. Bailey D, Faulkner R, Mackay H. Growth, physical activity, and bone mineral acquisition. Exerc Sport Sci Rev 1996;24:233-66.

2. Jouanny P, Guillemin F, Kuntz C, Jeandel C, Pourel J. Environmental and genetic factors affecting bone mass: similarity of bone density among members of health families. Arthritis Rheum 1995;38:61-7.

3. Theintz G, Buchs B, Rizzoli R, Slosman D, Clavien H, Sizonenko PC, et al. Longitudinal monitoring of bone mass accumulation in healthy adolescents: evidence for a marked reduction after 16 years of age at the levels of lumbar spine and femoral neck in female subjects. J Clin Endocrinol Metab 1992;5:1060-5.

4. Teegarden D, Lyle RM, Proulx WR, Johnston CC, Weaver CM. Previous milk consumption is associated with greater bone density in young women. Am J Clin Nutr 1999;69:1014-7.

5. Pocock NA, Eisman JA, Hopper JL, Yeates MG, Sambrook PN, S. E. Genetic determinants of bone mass in adults: A twin study. J Clin Invest 1991;80:706-10.

6. Lazaretti-Castro M. Why to evaluate bone mineral density in children and adolescents? J Pediatr 2004;80:439-40.

7. Kemper HC. Skeletal development during childhood and adolescence and the effects of physical activity. Pediatr Exerc Sci 2000;12:198-216.

8. Marcus R. Role of exercise in preventing and treating osteoporosis. Rheum Dis Clin North Am 2001;27:131-41, vi. Review.

9. Frost HM. Bone "mass" and the "mechanostat": a proposal. Anat Rec 1987;1:1-9.

10. Andreoli A, Monteleone M, Van Loan M, Promenzio L, Tarantino U, De Lorenzo A. Effects of different sports on bone density and muscle mass in highly trained athletes. Med Sci Sports Exerc 2001;33:507-11.

11. Lehtonen-Veromaa L, Mottonen T, Irjala K, Nuotio I, Leino A, Viikari J. A 1-Year prospective study on the relationship between physical activity, markers of bone metabolism, and bone acquisition in peripubertal girls. J Clin Endocrinol Metab 2000;85:3726-32.

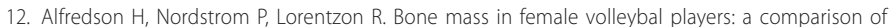
total and regional bone mass in female volleyball players an nonactive females. Calcif Tissue Int 1997;60:338-42.

13. Lee EJ, Long KA, Risser WL, Poindexter HB, Gibbons WE, Goldzieher J. Variations in bone status of contralateral and regional sites in young athletic women. Med Sci Sports Exerc 1995;27:1354-61.

14. Fehling PC, Alekel J, Clasey A, Rector A, Stillman RJ. A comparison of bone mineral densities among female athletes in impact loading and active loading sports. Bone 1995;17:205-10.

15. Creighton DL, Morgan AL, Boardley D, Brolinson PG. Weight-bearing exercise and markers of bone turnover in female athletes. J Appl Physiol 2001;90:565-70.

16. Giugliano R. Fundamentos de Nutrição. 2a. ed. Brasilia: Universa, 2000.

17. Pinheiro ABV, Lacerda EMA, Benzecry EH, Gomes MCS, Costa VM. Tabela para avaliação de consumo alimentar em medidas caseiras. 2a. ed. Rio de Janeiro: Atheneu, 1994.
18. Mazess R, Collick B, Trempe J, Barden H, Hanson J. Performance evaluation of a dual-energy x-ray bone densitometer. Calcif Tissue Int 1989;44:228-32.

19. Duke PM, Litt IF, Gross RT. Adolescents' self-assessment of sexual maturation. Pediatrics 1980;66:918-20.

20. Silva CC, Teixeira AS, Goldberg TBL. O esporte e suas implicações na saúde óssea de atletas adolescentes. Rev Bras Med Esporte 2003;9:426-32.

21. Cambraia AN, Pulcinelli AJ. Avaliação da composição corporal e da potência aeróbica em jogadoras de Voleibol de 13 a 16 anos de idade do Distrito Federal. Rev Bras Ciên e Mov 2002;10:43-8.

22. Fonseca RMC, França NM, Van Praagh E. Relationship Between Indicators of Fitness and Bone Density in Adolescent Brazilian Children. Pediatr Exerc Sci 2008;20:40-9.

23. Waltrick ACA, Duarte MFS. Estudo das características antropométricas de escolares de 7 a 17 anos - uma abordagem longitudinal mista e transversal. Rev Bras Cineantropometria \& Desempenho Humano 2000;2:17-30.

24. Filardo RD, Rodriguez-Añez CR, Pires Neto CS. Antropometria e composição corporal de jovens do sexo feminino entre 13 e 17 anos de idade. Rev Bras Cineantropometria \& Desempenho Humano 2000;2:66-71.

25. Almeida TA, Soares EA. Perfil dietético e antropométrico de atletas adolescentes de voleibol. Rev Bras Med Esporte 2003;9:191-7.

26. Bulhões JMA. Densidade mineral óssea em adolescentes femininas: Relação entre o sobrepeso e atividade física diária. Brasilia: Universidade Católica de Brasilia, 2005.

27. Escott-Strump S, Mahan KL. Krause: alimentos, nutrição \& dietoterapia. 11a. ed. São Paulo: Roca, 2005.

28. Health Nlo. Optimal calcium intake. Journal of the American Medical Association. 1994;272(24):1942-8.

29. Braggion GB, Matsudo SMM, Matsudo VKR. Consumo alimentar, atividade física e percepção da aparência corporal em adolescentes. Rev Bras Ciên e Mov. 2000;8:15-21.

30. Lerner BR, Lei DLM, Chaves SP, Freire RD. O Cálcio consumido por adolescentes de escolas públicas de Osasco. Rev Nutr 2000;13:57-63.

31. Loro ML, Sayre J, Roe TF, Goran MI, Kaufman FR, Gilsanz V. Early Identification of Children Predisposed to Low Peak Bone Mass and Osteoporosis Later in Life. J Clin Endocrinol Metab 2000;85:3908-18.

32. Snow-Harter C, Marcus R. Exercise, bone mineral density and osteoporosis. Exerc Sport Sci Rev. 1991;19:351-88.

33. Nordstrom P, Nordstrom G, Lorentzon R. Correlation of bone density to strength and physical activity in young men with a low or moderate level of physical activity. Calcif Tissue Int 1997;60:332-7.

34. Soderman K, Bergstrom E, Lorentzon R, Alfredson H. Bone mass and muscle strength in young female soccer players. Calcif Tissue Int 2000;67:297-303.

35. Pettersson U, Nordstrom P, Alfredson $\mathrm{H}$, Henriksson-Larsen K, Lorentzon R. Effect of high impact activity on bone mass and size in adolescent females: a comparative study between two different types of sports. Calcif Tissue Int 2000;67:207-17. 